\title{
Slingshot Nonsequential Double Ionization as a Gate to Anticorrelated Two-Electron Escape
}

\author{
G. P. Katsoulis, ${ }^{1}$ A. Hadjipittas, ${ }^{1}$ B. Bergues, ${ }^{2}$ M. F. Kling, ${ }^{2}$ and A. Emmanouilidou ${ }^{1}$ \\ ${ }^{1}$ Department of Physics and Astronomy, University College London, Gower Street, London WC1E 6BT, United Kingdom \\ ${ }^{2}$ Department of Physics, Ludwig-Maximilians-Universität Munich, Am Coulombwall 1, D-85748 Garching, Germany \\ and Max Planck Institute of Quantum Optics, Hans-Kopfermann-Strasse 1, D-85748 Garching, Germany
}

(Received 24 May 2018; revised manuscript received 21 August 2018; published 28 December 2018)

\begin{abstract}
At intensities below the recollision threshold, we show that recollision-induced excitation with one electron escaping fast after recollision and the other electron escaping with a time delay via a Coulomb slingshot motion is one of the most important mechanisms of nonsequential double ionization (NSDI), for strongly driven He at $400 \mathrm{~nm}$. Slingshot NSDI is a general mechanism present for a wide range of low intensities and pulse durations. Anticorrelated two-electron escape is its striking hallmark. This mechanism offers an alternative explanation of anticorrelated two-electron escape obtained in previous studies.
\end{abstract}

DOI: 10.1103/PhysRevLett.121.263203

Nonsequential double ionization (NSDI) in strong infrared laser fields is a fundamental process [1-23] accounted for by the three-step model [1]. First, one electron tunnel ionizes in the field-lowered Coulomb barrier and then accelerates in the laser field. This electron can return back to the core to recollide and transfer energy to a bound electron through different pathways. In the direct one, the energy transferred suffices for both electrons to ionize shortly after recollision. In the delayed pathway, the energy transferred ionizes one of the two electrons shortly after recollision. The other electron transitions to an excited state and ionizes later. It is generally accepted that recollisioninduced excitation with subsequent field ionization (RESI) [7,9] prevails the delayed pathway. In RESI, the excited electron ionizes at the field extrema, after recollision, assisted by the laser field. In the double delayed pathway, both electrons ionize later, following the energy transferred during recollision.

At intensities below the recollision threshold, in NSDI two electrons escaping opposite to each other along the laser-field direction-anticorrelated escape-has been studied intensely by experiment and theory alike. This pattern was found to prevail, but not substantially, over correlated two-electron escape. It was observed in NSDI of several atoms driven by intense (strongly driven) long duration pulses [24-31]. Multiple recollisions, in the context of RESI, were put forth to explain anticorrelated two-electron escape [24-28]. Electron-electron repulsion was also suggested as a possible explanation [30,31].

Here, we show that RESI does not necessarily prevail the delayed pathway, for strongly driven $\mathrm{He}$ at $400 \mathrm{~nm}$. We find a competing mechanism in the delayed pathway where the electron that ionizes later undergoes a slingshot motion due to the Coulomb interaction with the nucleus and the field. This Coulomb slingshot motion is similar to the well-known gravitational slingshot that alters the motion of a spacecraft around a planet. Moreover, we find that the electron undergoing a slingshot motion ionizes around the second extremum of the laser field after recollision. We label this mechanism slingshot NSDI. The nucleus has a small effect on RESI. In contrast, in slingshot NSDI the nucleus plays a decisive role with anticorrelated twoelectron escape being its striking hallmark. Slingshot NSDI is an alternative to the multiple recollisions mechanism for explaining anticorrelated two-electron escape.

We demonstrate slingshot NSDI in He driven by a nearsingle-cycle laser pulse at $5 \times 10^{14} \mathrm{~W} / \mathrm{cm}^{2}$ and $400 \mathrm{~nm}$. Further below, we discuss slingshot NSDI for other laser intensities and pulse durations as well. Kinematically complete experiments that employ carrier-envelope-phase-(CEP) controlled near-single-cycle pulses have been carried out for NSDI over a wide range of intensities [28,32-34]. The intensity of $5 \times 10^{14} \mathrm{~W} / \mathrm{cm}^{2}$ is below the recollision threshold. This corresponds to the maximum energy of the electron returning to the core, $3.17 \mathcal{E}_{0}^{2} /\left(4 \omega^{2}\right)$ [1], which is equal to $23.7 \mathrm{eV}$ at $5 \times 10^{14} \mathrm{~W} / \mathrm{cm}^{2}$, being equal to the energy needed to transition to the first excited state of the ion; $\mathcal{E}_{0}$ and $\omega$ are the strength and frequency of the field.

We employ a three-dimensional semiclassical model [35-37]. One electron (recolliding) tunnel ionizes through the field-lowered Coulomb barrier. We use the quantum mechanical Ammosov-Delone-Krainov formula to compute the tunnel-ionization rate [38,39]. The exit point of the recolliding electron is along the laser-field direction and is computed using parabolic coordinates [40]. The electron momentum is taken to be equal to zero along the laser field while the transverse one is given by a Gaussian distribution [38,39]. The initially bound electron is described by a microcanonical distribution [41]. 
We use a laser field of the form

$$
\overrightarrow{\mathcal{E}}(t)=\mathcal{E}_{0} \exp \left[-2 \ln 2\left(\frac{t}{\tau}\right)^{2}\right] \cos (\omega t+\phi) \hat{z}
$$

where $\phi$ is the CEP and $\tau=2$ fs is the full width at half maximum of the pulse duration in intensity. We employ atomic units, unless otherwise stated. The tunnel-ionization time $t_{0}$ is selected randomly in the time interval $[-2 \tau, 2 \tau]$. Once the initial conditions are specified at time $t_{0}$, the position and momentum of each electron are propagated classically in time. We do so using the three-body Hamiltonian of the two electrons with the nucleus kept fixed. All Coulomb forces and the interaction of each electron with the laser field are fully accounted for with no approximation. We also account for the Coulomb singularity by using regularized coordinates [42]. Our results for NSDI are obtained by taking into account CEPs that range from $\phi=0^{\circ}$ to $\phi=330^{\circ}$ in steps of $30^{\circ}$ and by averaging over these CEPs.

We identify the main pathways of energy transfer in each double ionization (DI) event. To do so, we compute the time difference between the recollision time $t_{\text {rec }}$ and the ionization time of each electron. We compare it with the time interval $t_{\text {diff }}$ where the electron pair potential energy undergoes a sharp change due to recollision. We find $t_{\text {diff }}$ to be roughly equal to $1 / 8$ laser cycle (T). We refer to the electron that, after recollision, ionizes first as electron 1, and the one ionizing last as electron 2. For each classical trajectory, the recollision time is defined as the time of minimum approach of the two electrons. It is identified by the maximum in the electron pair potential energy. The ionization time for each electron $t_{i}$ is defined as the time when the compensated energy $\left\{p_{x, i}^{2}+p_{y, i}^{2}+\right.$ $\left.\left[p_{z, i}-\mathcal{A}(t)\right]^{2}\right\} / 2-Z / r_{i}$ becomes positive and remains positive thereafter [43], with $i=1,2$ and $\mathbf{p}_{i}=p_{x, i} \hat{x}+$ $p_{y, i} \hat{y}+p_{z, i} \hat{z} ; \mathcal{A}(t)$ is the vector potential and $Z=2$. We list the conditions for a direct, delayed, or double delayed DI event in Table I. In the delayed pathway, the probability for electron 2 to be the recolliding electron increases with decreasing intensity.

At intensities above the recollision threshold, the direct pathway dominates. The two electrons escape mostly in the same direction along the laser field, with the final momentum of each electron being roughly equal to $-\mathcal{A}\left(t_{\text {rec }}\right)$.

TABLE I. Conditions for energy transfer DI pathways.

\begin{tabular}{lcc}
\hline \hline & $\Delta t_{1}=t_{1}-t_{\text {rec }}$ and $\Delta t_{2}=t_{2}-t_{\text {rec }}$ \\
\hline Direct & Delayed & Double delayed \\
\hline$\Delta t_{2}<t_{\text {diff }}$ & $\Delta t_{1}<t_{\text {diff }}$ & $\Delta t_{1}>t_{\text {diff }}$ \\
$t_{1}<t_{2}$ & $\Delta t_{2}>t_{\text {diff }}$ & $\Delta t_{2}>t_{\text {diff }}$ \\
\hline \hline
\end{tabular}

At intensities well below the recollision threshold, the delayed and double delayed pathways prevail. This is the case for He driven by a near-single-cycle laser pulse at $5 \times 10^{14} \mathrm{~W} / \mathrm{cm}^{2}$ and $400 \mathrm{~nm}$. We find that the two electrons escape overwhelmingly in opposite directions, see Fig. 1. In the double delayed pathway the two electrons escape with small momenta, see Fig. 1(b), whereas in the delayed pathway the momenta are larger [Fig. 1(c)]. To understand the anticorrelation pattern, we focus on the delayed pathway and its contributing mechanisms.

In the delayed pathway, electron 1 escapes with momentum roughly equal to $-\mathcal{A}\left(t_{1} \approx t_{\text {rec }}\right)$, which is mostly large, since recollision occurs around a zero of the field. In RESI, electron 2 ionizes at a time $t_{2}$ around an extremum of the field. It does so primarily due to the laser field, and, thus, has a small final momentum, $-\mathcal{A}\left(t_{2}\right)$, along the $\hat{z}$ axis; see Fig. 1(c). In the new mechanism, which we refer to as slingshot NSDI, the final momentum of electron 2 is determined both by the Coulomb field and the laser field and it has mostly large magnitude; see Fig. 1(c). Moreover, unlike RESI, in slingshot NSDI the two electrons escape only in opposite directions.

Next, we describe slingshot NSDI using a representative delayed pathway event for $\phi=0^{\circ}$ that encapsulates the main features of the new mechanism. Below, the description of slingshot NSDI and RESI applies to all CEPs where the recolliding electron tunnel ionizes from a minimum of the laser field. A similar description applies to CEPs where tunnel ionization occurs from a maximum of the field. The difference is that some of the quantities plotted in Fig. 2 would be reflected with respect to the time axis. For simplicity, in Fig. 2, we focus on the times after recollision. Electron 1 (green line) ionizes in the time interval $[0,0.5] \mathrm{T}$ with a positive momentum, mostly large as for all delayed events.

In slingshot NSDI, electron 2 (black line) initially moves away from the nucleus along the $+\hat{z}$ axis. However, it soon returns and undergoes a close encounter with the nucleus at time $t_{2}^{\text {ret. }}$, see $r_{z, 2}$ as a function of time in Fig. 2(a1), where $\mathrm{r}_{z, 2}$ is the $z$ component of the position vector $\mathbf{r}_{2}$. Indeed, $r_{z, 2}$ has a very small negative value at time $t_{2}^{\text {ret. }}$. This return of electron 2 to the nucleus leads to a slingshot motion in

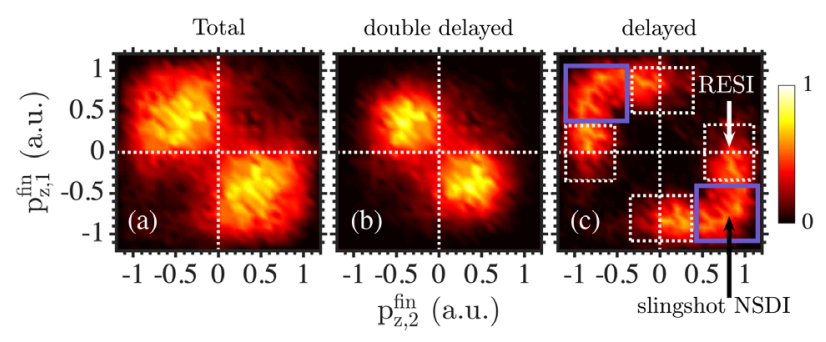

FIG. 1. Symmetrized correlated electron momenta normalized to peak value for (a) all DI, (b) double delayed, and (c) delayed events for He driven by a near-single-cycle pulse at $5 \times 10^{14} \mathrm{~W} / \mathrm{cm}^{2}$ and $400 \mathrm{~nm}$. 

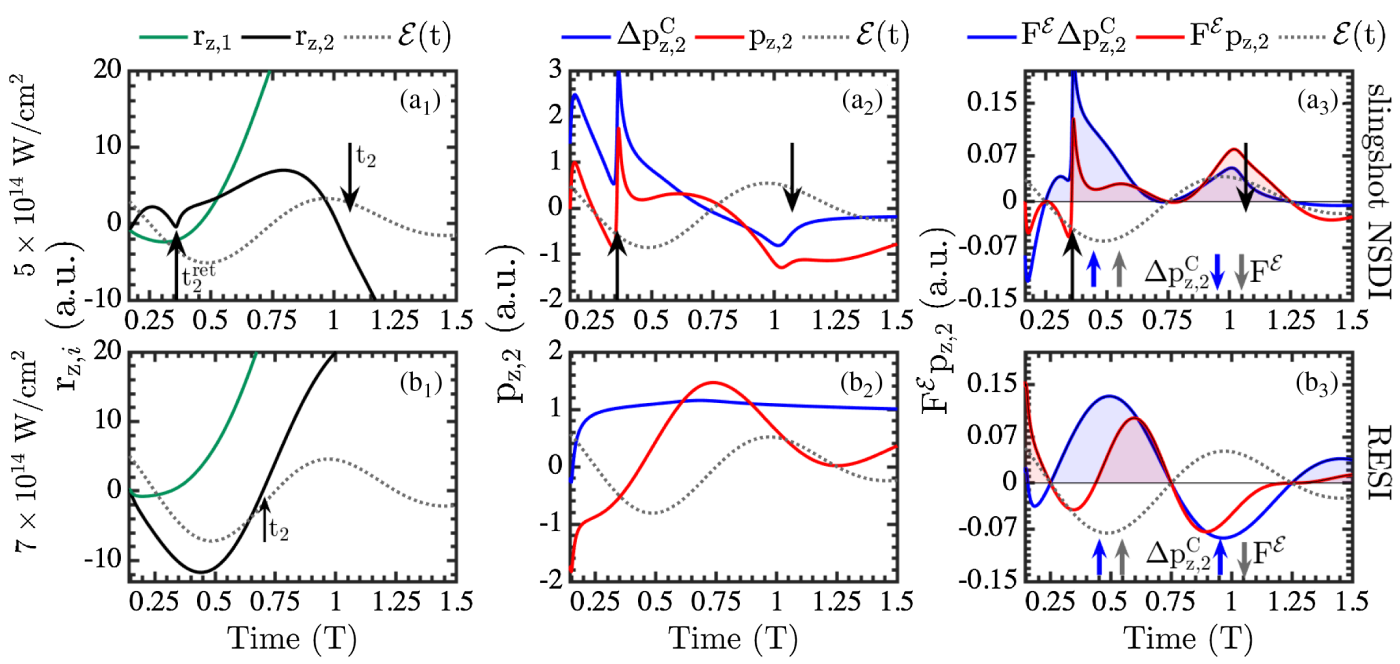

FIG. 2. Slingshot NSDI at $5 \times 10^{14} \mathrm{~W} / \mathrm{cm}^{2}$ (a) and RESI at $7 \times 10^{14} \mathrm{~W} / \mathrm{cm}^{2}$ (b) for He driven by a near-single-cycle pulse at $400 \mathrm{~nm}$. Plotted as a function of time are (a1),(b1) $r_{z, 1}$ and $r_{z, 2}$, (a2),(b2) $p_{z, 2}$ and $\Delta p_{z, 2}^{C}$, and (a3),(b3) $F^{\mathcal{E}} p_{z, 2}$ and $F^{\mathcal{E}} \Delta p_{z, 2}^{C}$. CS is enclosed by an up and down black arrow, which represent $p_{z, 2}$ being along the $+\hat{z}$ axis and $-\hat{z}$ axis, respectively, at the start and end of CS. The beginning of the time axis is $t_{\text {rec }}$.

the time interval $t_{2}^{\text {ret }}$ to $t_{2}$, denoted by black arrows in Figs. 2(a1)-2(a3). During this motion electron 2 remains close to the nucleus due to the comparable magnitude of the attractive Coulomb force and the force of the laser field. Moreover, in Coulomb slingshot (CS), the momentum of electron 2 changes significantly. It has a large positive value at $t_{2}^{\text {ret }}$, which has the same sign as the final momentum of electron 1, to a large negative value at the time electron 2 ionizes, $t_{2}$; see red line in Fig. 2(a2). Hence, CS results in electron 2 escaping opposite to electron 1 along the laser field. The effect of the nucleus has previously been addressed in the context of strongly driven clusters [44].

For most slingshot-NSDI events electron 2 ionizes around the second extremum of the field in the time interval $[0.75,1.25] \mathrm{T}$. To explain why this is the case, we employ the energy of electron 2 . Shortly after recollision, at time $t_{\text {init }}=t_{\text {rec }}+t_{\text {diff }}$, the repulsive force between the two electrons is significantly smaller than during recollision. Hence, after this time, the energy of electron 2 changes due to the work done mainly by the field as follows:

$$
H(t)=\frac{p_{2}\left(t_{\text {init }}\right)^{2}}{2}-\frac{Z}{r_{2}\left(t_{\text {init }}\right)}+\int_{t_{\text {init }}}^{t} F^{\mathcal{E}} p_{z, 2} d t^{\prime}
$$

where $F^{\mathcal{E}}(t)=-\mathcal{E}(t)$ is the force from the laser field and $F^{\mathcal{E}} p_{z, 2}$ is the rate of change of the energy of electron 2 . During CS, the close encounter of electron 2 with the nucleus at $t_{2}^{\text {ret }}$ takes place past a zero of the laser field. At this time both the momentum of electron 2 and the force from the laser field point along the $+\hat{z}$ axis. Roughly half a laser cycle later, in the time interval $[0.75,1.25] \mathrm{T}$, the slingshot motion is concluded with both the momentum of electron 2 and the force of the laser field pointing along the $-\hat{z}$ axis. Thus, during CS, $F^{\mathcal{E}} p_{z, 2}$ is mostly positive in the first half cycle $[0.25,0.75] \mathrm{T}$ and the second one $[0.75$, 1.25] $\mathrm{T}$ after recollision; see red shaded area in Fig. 2(a3). This is the reason electron 2 gains sufficient energy to ionize around the second extremum of the field after recollision.

Next, we identify the main reason that the rate of change of the energy of electron 2 is positive during CS. We express the total momentum of electron 2 as the sum of the momentum changes due to the interaction with the nucleus and electron $1, \Delta p_{z, 2}^{C}$, and with the laser field, $\Delta p_{z, 2}^{\mathcal{E}}$, as follows:

$p_{z, 2}(t)=p_{z, 2}\left(t_{0}\right)+\Delta p_{z, 2}^{C}\left(t_{0} \rightarrow t\right)+\Delta p_{z, 2}^{\mathcal{E}}\left(t_{0} \rightarrow t\right)$,

$\Delta p_{z, 2}^{C}(t)=\int_{t_{0}}^{t}\left(\frac{-Z r_{z, 2}}{\left|r_{2}\right|^{3}}+\frac{r_{z, 2}-r_{z, 1}}{\left|\mathbf{r}_{2}-\mathbf{r}_{1}\right|^{3}}\right) d t^{\prime}$,

$\Delta p_{z, 2}^{\mathcal{E}}(t)=\mathcal{A}(t)-\mathcal{A}\left(t_{0}\right)$.

For delayed events, the repulsive force between the two electrons is roughly zero shortly after recollision, thus contributing only a constant term to $\Delta p_{z, 2}^{C}$. We plot the momentum change due to the nucleus as well as the total momentum of electron 2 in Fig. 2(a2). It is clear that, during CS, the sharp change of the total momentum of electron 2 is mainly due to the term $\Delta p_{z, 2}^{C}$. Hence, the attractive force of the nucleus causes the main change in the total momentum of electron 2. Combining Eqs. (2) and (3), we also plot in Fig. 2(a3) the contribution of the nucleus, $F^{\mathcal{E}} \Delta p_{z, 2}^{C}$ (blue line), to the rate of change of the energy of electron 2 , $F^{\mathcal{E}} p_{z, 2}$ (red line). We find that, during CS, the contribution of the nucleus (blue shaded area) causes this rate to be 
mostly positive (red shaded area). The contribution of the field, $F^{\mathcal{E}} \Delta p_{z, 2}^{\mathcal{E}}$ (not shown), is also mostly positive during the last part of CS.

We find that the dominant mechanism of the delayed pathway is slingshot NSDI at $5 \times 10^{14} \mathrm{~W} / \mathrm{cm}^{2}$ and RESI at $7 \times 10^{14} \mathrm{~W} / \mathrm{cm}^{2}$. We illustrate RESI in Fig. 2(b). In contrast to slingshot NSDI, in RESI the momentum change of electron 2 due to the nucleus is almost a constant after recollision; see blue line in Fig. 2(b2). Moreover, the strength of the laser field $F^{\mathcal{E}}$ is larger at the higher intensity. Thus, in the first half cycle, the rate of change of the energy of electron $2, F^{\mathcal{E}} p_{z, 2}$ in Fig. 2(b3), has larger positive values at $7 \times 10^{14} \mathrm{~W} / \mathrm{cm}^{2}$ rather than at $5 \times 10^{14} \mathrm{~W} / \mathrm{cm}^{2}$. Hence, electron 2 ionizes mostly around the first extremum in RESI at $7 \times 10^{14} \mathrm{~W} / \mathrm{cm}^{2}$. The two mechanisms are compared in detail in Supplemental Material [45].

Slingshot NSDI for He driven by a 2 fs laser pulse at $5 \times 10^{14} \mathrm{~W} / \mathrm{cm}^{2}$ and $400 \mathrm{~nm}$ accounts for roughly $40 \%$ of the delayed pathway. In Fig. 3 we demonstrate that slingshot NSDI is a general mechanism that significantly contributes to the delayed pathway of NSDI for a wide range of laser intensities and pulse durations for $\mathrm{He}$ at $400 \mathrm{~nm}$. First, we keep the pulse duration constant and increase the intensity from $5 \times 10^{14}$ to $7 \times 10^{14} \mathrm{~W} / \mathrm{cm}^{2}$. Besides the delayed pathway, another important pathway is the double delayed one at $5 \times 10^{14} \mathrm{~W} / \mathrm{cm}^{2}$ and the direct one at $7 \times 10^{14} \mathrm{~W} / \mathrm{cm}^{2}$. The contribution of slingshot NSDI to the delayed pathway is significant for all these pulse parameters; however, it decreases with increasing intensity; see Fig. 3. This is consistent with the increasing contribution of RESI to the delayed pathway.

Keeping the intensity constant, we change the pulse duration from 2 to $6 \mathrm{fs}$. We find that the contribution of slingshot NSDI to the delayed pathway decreases with increasing pulse duration; see Fig. 3. For a longer pulse the force from the laser field is larger at a given extremum of the field. Hence, we conjecture that it is possible that, while CS still occurs, CS is not as pronounced and the last electron finally ionizes at subsequent half cycles also due to the contribution of the field to the rate of change of the energy of electron $2, F^{\mathcal{E}} \Delta p_{z, l}^{\mathcal{E}}$. Moreover, a longer pulse

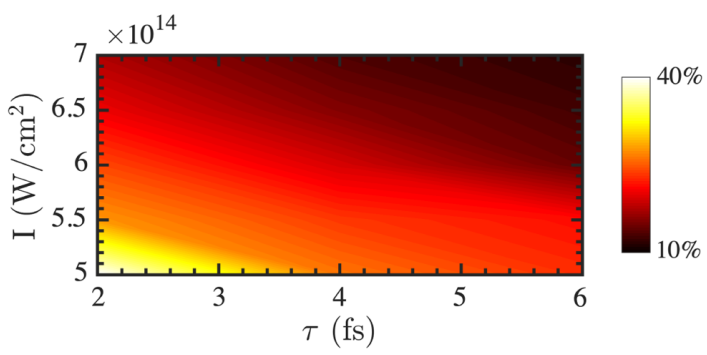

FIG. 3. Contribution of slingshot NSDI to the delayed pathway as a function of laser intensity in steps of $0.5 \times 10^{14} \mathrm{~W} / \mathrm{cm}^{2}$ and of pulse duration in steps of 2 fs. allows for a more complicated interaction of electron 2 with the nucleus. This can result in electron 2 escaping in the same or opposite direction from the first electron. The above arguments are consistent with our finding that in the delayed pathway the anticorrelation pattern is more pronounced for shorter compared to longer pulses.

For He driven at $400 \mathrm{~nm}$, two experiments are most relevant to the work presented here [16,23]. In Ref. [16], excellent agreement was found between experiment and fully $a b$ initio quantum mechanical calculations for electron energy distributions of doubly ionized He driven by a long duration laser pulse [16]. For the latter observables, using the model described here, we achieved excellent agreement with fully ab initio quantum mechanical calculations [36]; the latter were performed by the same theoretical group as in Ref. [16]. Recently, a study of a kinematically complete experiment for $\mathrm{He}$ driven by a long duration laser pulse at $400 \mathrm{~nm}$ and intensities of $(3.5-5.7) \times 10^{14} \mathrm{~W} / \mathrm{cm}^{2}$ was submitted and published [23]. In this latter work, the correlated electron momenta are obtained. As the intensity increases from $3.5 \times 10^{14}$ to $5.7 \times 10^{14} \mathrm{~W} / \mathrm{cm}^{2}$, a transition from anticorrelated plus correlated to mostly correlated two-electron escape is observed. We also find such a transition taking place for $\mathrm{He}$ driven at $400 \mathrm{~nm}$, as the intensity increases from $5 \times 10^{14}$ to $7 \times 10^{14} \mathrm{~W} / \mathrm{cm}^{2}$, for 2, 4, and 6 fs laser pulses; see Ref. [45].

To guide experiments, we identify favorable laser parameters for observing an anticorrelated two-electron escape mostly due to slingshot NSDI. In the direct pathway the two electrons escape overwhelmingly in the same direction, while in the double delayed they escape mostly in opposite directions along the laser field. Thus, to observe slingshot NSDI, the contribution to NSDI of the double delayed pathway has to be small. We find that for the currently considered low intensities, the contribution of the double delayed pathway is smaller for the shorter duration laser pulses. We have also shown that, for low intensities, slingshot NSDI contributes most to the delayed pathway for short pulse durations. Given the above, it is essential that a short pulse duration is employed for observing slingshot NSDI.

In conclusion, slingshot NSDI is an important mechanism of the delayed pathway and of NSDI for a range of intensities and pulse durations for He driven at $400 \mathrm{~nm}$. In slingshot NSDI, following re collision, the electron that ionizes last undergoes a Coulomb slingshot within roughly half a laser cycle that changes sharply the direction of the electron momentum along the laser field. This electron has a large momentum that points in the same direction as the force from the laser field both at the start and at the end of CS. Hence, during CS the laser field supplies sufficient energy to this electron leading to its ionization around the second extremum of the field. The hallmark of slingshot NSDI is that the two electrons escape in opposite directions along the laser field. We expect slingshot NSDI to be a 
significant mechanism in NSDI of $\mathrm{He}$ driven at wavelengths other than $400 \mathrm{~nm}$, and to be present in other atoms and molecules.

A. E. acknowledges the EPSRC Grant No. N031326 and the use of the computational resources of Legion at UCL. M.F.K acknowledges support by the German Research Foundation (DFG) via the Cluster of Excellence: Munich Centre for Advanced Photonics (MAP), and by the European Union (EU) via the European Research Council (ERC) grant ATTOCO.

[1] P. B. Corkum, Phys. Rev. Lett. 71, 1994 (1993).

[2] A. l'Huillier, L. A. Lompre, G. Mainfray, and C. Manus, Phys. Rev. A 27, 2503 (1983).

[3] P. Lambropoulos, Phys. Rev. Lett. 55, 2141 (1985).

[4] B. Walker, B. Sheehy, L. F. DiMauro, P. Agostini, K. J. Schafer, and K. C. Kulander, Phys. Rev. Lett. 73, 1227 (1994).

[5] S. F. J. Larochelle, A. Talebpour, and S. L. Chin, J. Phys. B 31, 1201 (1998).

[6] R. Moshammer, B. Feuerstein, W. Schmitt, A. Dorn, C. D. Schröter, J. Ullrich, H. Rottke, C. Trump, M. Wittmann, G. Korn, K. Hoffmann, and W. Sandner, Phys. Rev. Lett. 84, 447 (2000).

[7] R. Kopold, W. Becker, H. Rottke, and W. Sandner, Phys. Rev. Lett. 85, 3781 (2000).

[8] T. Weber, H. Giessen, M. Weckenbrock, G. Urbasch, A. Staudte, L. Spielberger, O. Jagutzki, V. Mergel, M. Vollmer, and R. Dörner, Nature (London) 405, 658 (2000).

[9] B. Feuerstein, R. Moshammer, D. Fischer, A. Dorn, C. D. Schröter, J. Deipenwisch, J. R. C. Lopez-Urrutia, C. Höhr, P. Neumayer, J. Ullrich, H. Rottke, C. Trump, M. Wittmann, G. Korn, and W. Sandner, Phys. Rev. Lett. 87, 043003 (2001).

[10] R. Dörner, T. Weber, M. Weckenbrock, A. Staudte, M. Hattass, H. Schmidt-Böcking, R. Moshammer, and J. Ullrich, Adv. At. Mol. Opt. Phys. 48, 1 (2002).

[11] J. Ullrich, R. Moshammer, A. Dorn, R. Dörner, L. P. H. Schmidt, and H. Schmidt-Böcking, Rep. Prog. Phys. 66, 1463 (2003).

[12] E. Eremina, X. Liu, H. Rottke, W. Sandner, M. G. Schätzel, A. Dreischuh, G. G. Paulus, H. Walther, R. Moshammer, and J. Ullrich, Phys. Rev. Lett. 92, 173001 (2004).

[13] A. Rudenko, K. Zrost, B. Feuerstein, V. L. B. de Jesus, C. D. Schröter, R. Moshammer, and J. Ullrich, Phys. Rev. Lett. 93, 253001 (2004).

[14] P. J. Ho, R. Panfili, S. L. Haan, and J. H. Eberly, Phys. Rev. Lett. 94, 093002 (2005).

[15] A. Becker, R. Dörner, and R. Moshammer, J. Phys. B 38, S753 (2005).

[16] J. S. Parker, B. J. S. Doherty, K. T. Taylor, K. D. Schultz, C. I. Blaga, and L. F. DiMauro, Phys. Rev. Lett. 96, 133001 (2006).

[17] A. Staudte, C. Ruiz, M. Schöffler, S. Schössler, D. Zeidler, T. Weber, M. Meckel, D. M. Villeneuve, P. B. Corkum, A. Becker, and R. Dörner, Phys. Rev. Lett. 99, 263002 (2007).
[18] A. Rudenko, V. L. B. de Jesus, T. Ergler, K. Zrost, B. Feuerstein, C. D. Schröter, R. Moshammer, and J. Ullrich, Phys. Rev. Lett. 99, 263003 (2007).

[19] K. T. Taylor, J. S. Parker, D. Dundas, and K. J. Meharg, J. Mod. Opt. 54, 1959 (2007).

[20] A. N. Pfeiffer, C. Cirelli, M. Smolarski, R. Dörner, and U. Keller, Nat. Phys. 7, 428 (2011).

[21] K. Henrichs, M. Waitz, F. Trinter, H. Kim, A. Menssen, H. Gassert, H. Sann, T. Jahnke, J. Wu, M. Pitzer, M. Richter, M. S. Schöffler, M. Kunitski, and R. Dörner, Phys. Rev. Lett. 111, 113003 (2013).

[22] Y. Chen, Y. Zhou, Y. Li, M. Li, P. Lan, and P. Lu, J. Chem. Phys. 144, 024304 (2016).

[23] K. Henrichs, S. Eckart, A. Hartung, D. Trabert, K. Fehre, J. Rist, H. Sann, M. Pitzer, M. Richter, H. Kang, M. S. Schöffler, M. Kunitski, T. Jahnke, and R. Dörner, Phys. Rev. A 98, 043405 (2018).

[24] Y. Liu, S. Tschuch, A. Rudenko, M. Dürr, M. Siegel, U. Morgner, R. Moshammer, and J. Ullrich, Phys. Rev. Lett. 101, 053001 (2008).

[25] S. L. Haan, Z. S. Smith, K. N. Shomsky, and P. W. Plantinga, J. Phys. B 41, 211002 (2008).

[26] D. F. Ye and J. Liu, Phys. Rev. A 81, 043402 (2010).

[27] X. Ma, Y. Zhou, and P. Lu, Phys. Rev. A 93, 013425 (2016).

[28] M. Kübel, K. J. Betsch, N. G. Kling, A. S. Alnaser, J. Schmidt, U. Kleineberg, Y. Deng, I. Ben-Itzhak, G. G. Paulus, T. Pfeifer, J. Ullrich, R. Moshammer, M. F. Kling, and B. Bergues, New J. Phys. 16, 033008 (2014).

[29] Y. Zhou, Q. Liao, and P. Lu, Phys. Rev. A 80, 023412 (2009).

[30] Y. Zhou, C. Huang, and P. Lu, Phys. Rev. A 84, 023405 (2011).

[31] D. I. Bondar, G. L. Yudin, W. K. Liu, M. Y. Ivanov, and A. D. Bandrauk, Phys. Rev. A 83, 013420 (2011).

[32] N. G. Johnson, O. Herrwerth, A. Wirth, S. De, I. Ben-Itzhak, M. Lezius, B. Bergues, M. F. Kling, A. Senftleben, C. D. Schröter, R. Moshammer, J. Ullrich, K. J. Betsch, R. R. Jones, A. M. Sayler, T. Rathje, K. Rühle, W. Müller, and G. G. Paulus, Phys. Rev. A 83, 013412 (2011).

[33] N. Camus, B. Fischer, M. Kremer, V. Sharma, A. Rudenko, B. Bergues, M. Kübel, N. G. Johnson, M. F. Kling, T. Pfeifer, J. Ullrich, and R. Moshammer, Phys. Rev. Lett. 108, 073003 (2012).

[34] B. Bergues, M. Kübel, N. G. Johnson, B. Fischer, N. Camus, K. J. Betsch, O. Herrwerth, A. Senftleben, A. M. Sayler, T. Rathje, T. Pfeifer, I. Ben-Itzhak, R. R. Jones, G. G. Paulus, F. Krausz, R. Moshammer, J. Ullrich, and M. F. Kling, Nat. Commun. 3, 813 (2012).

[35] A. Emmanouilidou, Phys. Rev. A 78, 023411 (2008).

[36] A. Emmanouilidou, J. S. Parker, L. R. Moore, and K. T. Taylor, New J. Phys. 13, 043001 (2011).

[37] A. Chen, M. Kübel, B. Bergues, M. F. Kling, and A. Emmanouilidou, Sci. Rep. 7, 7488 (2017).

[38] L. D. Landau and E. M. Lifshitz, Quantum Mechanics (Pergamon Press, New York, 1977).

[39] N. B. Delone and V. P. Krainov, J. Opt. Soc. Am. B 8, 1207 (1991).

[40] B. Hu, J. Liu, and S. G. Chen, Phys. Lett. A 236, 533 (1997). 
[41] R. Abrines and I. C. Percival, Proc. Phys. Soc. London 88, 861 (1966).

[42] P. Kustaanheimo and E. Stiefel, J. Reine Angew. Math. 1965, 204 (1965).

[43] J. G. Leopold and I. C. Percival, J. Phys. B 12, 709 (1979).
[44] U. Saalmann and J. M. Rost, Phys. Rev. Lett. 100, 133006 (2008).

[45] See Supplemental Material at http://link.aps.org/ supplemental/10.1103/PhysRevLett.121.263203 for a comparison of slingshot NSDI and RESI and correlated electron momenta. 